\title{
A New Flexible Photogrammetry Instrumentation for Estimating Wing Deformation in Airbus
}

\author{
Quentin Demoulin ${ }^{1,2}$, François Lefebvre-Albaret ${ }^{1}$, Adrian Basarab ${ }^{3}$, Denis Kouamé3, \\ and Jean-Yves Tourneret ${ }^{2}$ \\ ${ }^{1}$ Airbus, 316 route de Bayonne, 31060 Toulouse, France, \\ 2 IRIT/ENSEEIHT/Tésa, University of Toulouse, 31071 Toulouse, France \\ 3 IRIT UMR CNRS 5505, University of Toulouse, Université Paul Sabatier, CNRS, France \\ email: quentin.demoulin@airbus.com
}

\begin{abstract}
:
As part of aircraft certification and optimization, wing bending and twist measurements are performed under various load cases (aircraft weight, speed, angle of attack, etc.) to validate and improve wing deformation models. Since these measurements are acquired during flight, their analysis requires to face strong environmental constraints. Indeed, the highly varying luminosity conditions, the presence of possible reflections or shadows, the vibrations and the deformations of the entire aircraft, are strong constraints that need to be considered carefully.

Current approaches applied in Airbus are based on inertial measurement units installed inside the wing, or on photogrammetry-based solutions using calibrated sensors and retro-reflective targets located on the wings. These methods are not only highly intrusive, but also require time-consuming installation, calibration phases and dedicated flights to produce only sparse measurements. Moreover, the use of reflective targets on the wing has an impact on the wing aerodynamic, which should be avoided.

In this paper, we investigate a new method for estimating wing deformations. This method adapts a photogrammetry approach classically used for reconstructing buildings or art structures to the aircraft environment. To this aim, we propose to use synchronous videos from high resolution cameras, which can be easily installed on the aircraft windows and on the vertical stabilizer. Appropriate features are extracted from the images acquired by these cameras, related to wing joints or reference points located on the aircraft wing. The system uses these features to autonomously recalibrate itself at each frame and provide a dense 3D reconstruction of the wing in the aircraft reference coordinate system. Some experiments conducted on real data acquired on Airbus aircrafts show that the proposed estimation method provide promising results.
\end{abstract}

Key words: Photogrammetry, Wing Deformation, Flight Tests, A350

\section{Introduction}

Theoretical models of aircraft deformations are developed to predict wing fatigue and behavior in all flight conditions, simulating the wing deformations for varying scenarii depending for example on aircraft weight, speed, or angle of attack. These models are built on the Finite Element Method (FEM) reflecting the mechanical properties of the aircraft. However, theoretical models may slightly differ from the real aircraft behavior. Therefore, in aircraft certification procedures, it is necessary to measure the 3D deformations of wings to assess and further correct the models. This paper considers the specific case of wing deformations, which is important for many practical applications.

First of all, one could instrument the wing of interest to acquire the measurements. However, placing sensors inside the wing requires difficult installation, and provides only few measurement points. Another solution would be to install sensors on the wing surface, but this operation should be conducted carefully, by avoiding to introduce disturbances in the wing aerodynamic properties. For these reasons, we consider in this study a non-contact 3D measurement method, based on multiple view photogrammetry form video cameras. It is worth mentioning that LIDAR (Light Detection and Ranging) reconstruction is also a potential good candidate for the problem addressed in this work.

The use of cameras for wing deformation estimation faces several constraints. On the one hand, camera positioning is restricted presently since the only available positions are on aircraft 
windows and on the aircraft rear stabilizer. Moreover, due to the geometry of the aircraft, cameras on windows with grazing views of the wing, should be close enough to guarantee a good overlap between images, which is not ideal for photogrammetry triangulation. Indeed, the optimal distance between cameras should be close to the distance between each camera and the observed object. Finally, we must also take into account that cameras will slightly move during the flight as the whole aircraft is deforming.

On the other hand, our system should be robust to varying environmental conditions. Indeed, luminosity variations during the flight, potential shadows or reflections on the wing surface strongly impact the 3D reconstruction method. For instance, Fig. 1 illustrates the impact of luminosity variations on some real images, showing the variability of the observed images.

This paper is structured as follows. Section 1 presents the current methods applied in Airbus to provide the wing deformation measurements. The proposed new photogrammetry approach is introduced in Section 3. Results obtained on real Aircraft measurements are reported in Section 4. Conclusion and perspectives are finally reported in Section 5.

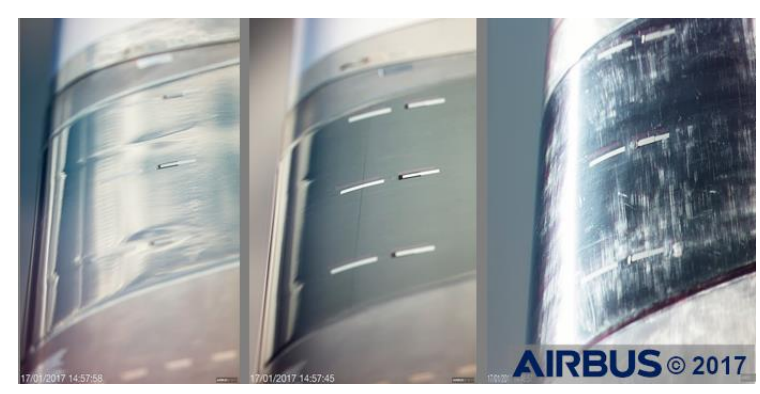

Fig. 1. Several views of the same wing leading edge under various illuminations.

\section{Current Reconstruction Method}

To estimate wing twist and bending, recent aircraft used in Airbus flight tests consider inertial motion units (IMU) installed inside the wing. These systems can deliver real time and accurate measurement of the wing bending and twist with respect to a reference inertial unit at the center of the aircraft. However, IMUs are not always available, and they only produce sparse measurements. Indeed, only a few IMUs can be installed inside the wing as they require difficult installation. Therefore, for some specific needs, Airbus also uses a photogrammetry approach.

\subsection{Photogrammetry}

The photogrammetry system used by Airbus to estimate wing deformation is based on retro-reflective targets stuck orthogonally to the wing surface, observed by cameras installed on windows. The estimation approach is similar to the studies presented [1] and [2] (see Fig. 2 for illustration). To limit distortions, standard windows in front of the cameras are replaced by metrological ones. During flight, cameras are triggered synchronously with flashes, illuminating the targets to easily and accurately detect targets. Finally, the accurate position of targets and cameras is initialized through a long calibration phase, and cameras are attached such that that their locations remain fixed with respect to the aircraft.

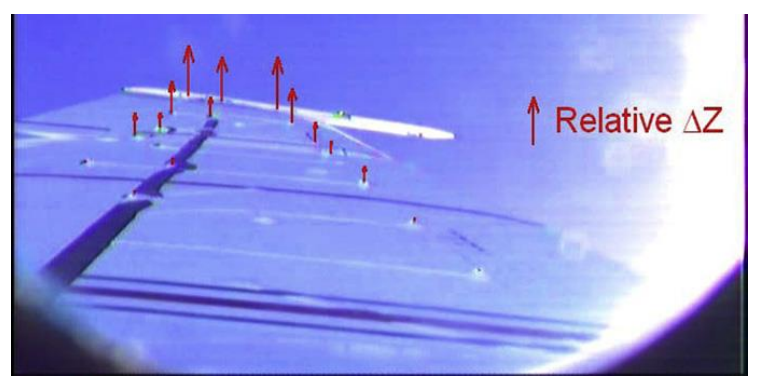

Fig. 2. Example of an aircraft wing imaged using a photogrammetry system (from [2]).

Though this system provides very satisfying results during tests, it has several drawbacks. First, the use of protuberant targets on the wing induces aerodynamic disturbances, which can alter the aerodynamic properties of the wing and reduce the flight domain of the aircraft, thus enforcing the need for dedicated fights. Secondly, to reduce the camera motions during flight, the mechanical fixations of the cameras are intrusive and not easy to install. Another drawback is the time consuming calibration step required for the system, which could be lightened. Finally, the method provides only sparse measurements associated with target locations, and the necessary time to recharge our flashes does not allow us to record several images per second.

\subsection{Other Studies}

Other investigations in the domain of contactless $3 \mathrm{D}$ reconstruction were perform in Airbus. In this field, we can mention the IPCT (image pattern correlation technique) which was tested on aircraft A380 [15] and A350. Using pairs of cameras, this technique combines image correlation and photogrammetry algorithms to recover the 3D shape and deformations of a surface. To capture image correlations, the method relies on the presence of image texture. Thus stickers of random pattern are stuck on the wing surface (see Fig. 4). Deflections of the surface are calculated using a simplified deformation model of the wing as function of a normal load under static conditions. 
Another explored method is based on deflectometry, which was tested in the A340 BLADE (Breakthrough Laminar Aircraft Demonstrator in Europe) project [3].The principle of this approach is to consider the reflection of a known pattern on a surface, and recover the local surface deformations from the reflected pattern deformations. To use this approach, strong assumptions on surface properties must be made (geometry, reflecting properties), which is only feasible within very specific applications. In the frame of the BLADE project, a pattern was painted on the wing edge, reflected on the wing, and reflections were observed by cameras installed at the wing tip (see Fig. 3). To ensure good reflection of the pattern, a specific paint was used on the wing surface.

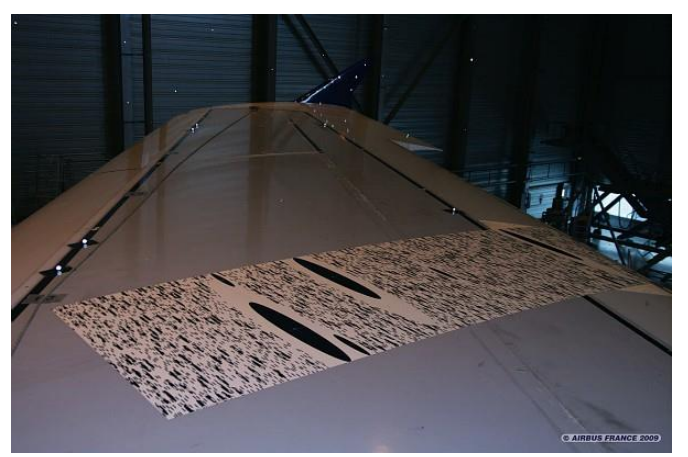

Fig. 4. Example of pattern stripe installed for IPCT tests on A380 in 2009.

Note that both methods gave interesting results, but the required installations are intrusive and onerous, and hence should be avoided.

\section{Proposed Photogrammetry Approach}

In this Section, we describe a new photogrammetry approach that cope with the principle drawbacks of the previous methods. This method operates a set of cameras installed at window positions and another camera located on the vertical stabilizer. The wing deformations are then estimated by recovering the $3 \mathrm{D}$ coordinates of points located on the wing surface, using a generic photogrammetry method adapted to the specific case of flight tests.

\subsection{Photogrammetry Principle}

The basic concept of photogrammetry is to recover the 3D position of points from their observations on images. To better understand its concept, some mathematical principles are recalled.

We observe a set of 3D points $\left(\boldsymbol{X}_{i}\right)_{=1, \ldots, N}$, with $N$ the number of points, using a set of $M$ cameras (depicted in Fig. 5). Each camera has its own coordinate system with respect to that of the aircraft. The 3D points are expressed in the aircraft coordinate system. To transfer the points from

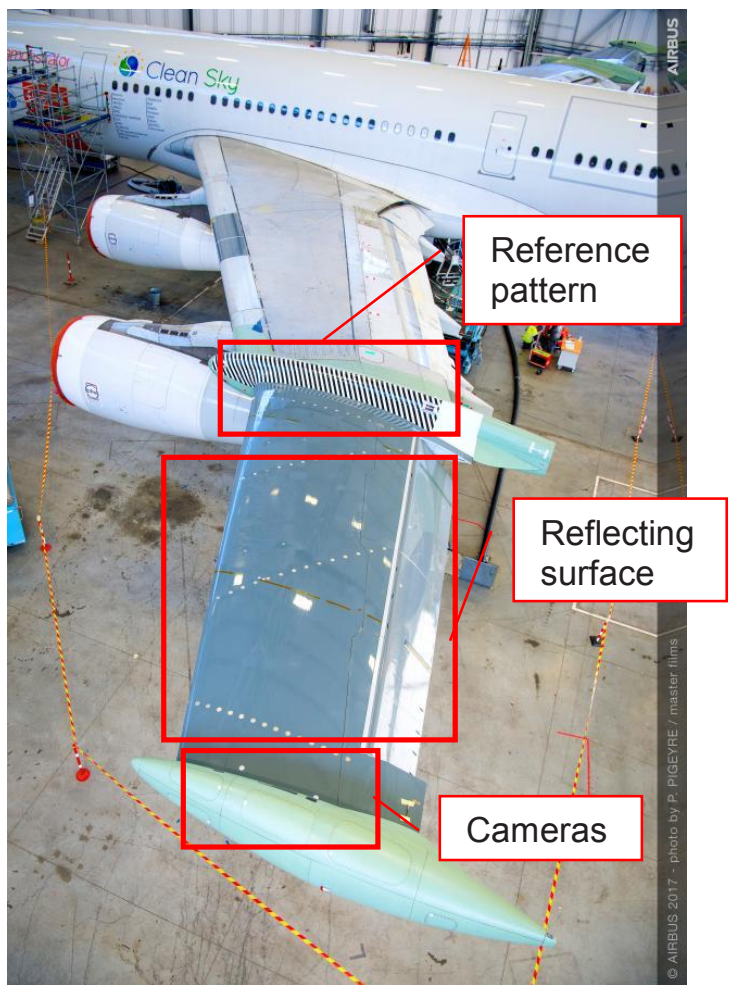

Fig. 3. BLADE wing with deflectometry installation.

this system to the camera one, we use the camera transfer matrices $\left(\boldsymbol{P}^{j}\right)_{j=1, \ldots, M}$, which represent the extrinsic parameters of the $M$ camera (rotations and translations). Then 3D points are projected from camera to image coordinates using the matrices $\left(\boldsymbol{K}^{j}\right)_{j=1, \ldots, M}$, representing the camera intrinsic parameters (focal, sensor center, pixel size). These matrices can be estimated from a calibration phase. The resulting 2D points can then be expressed as a function of the 3D points and camera matrices:

$$
\boldsymbol{x}_{i}^{j}=f\left(\boldsymbol{X}_{i}, \boldsymbol{P}^{j}, \boldsymbol{K}^{\boldsymbol{j}}\right)
$$

where $\boldsymbol{x}_{i}^{j}$ and $\boldsymbol{K}^{j}$ are known quantities. To recover the $3 \mathrm{D}$ points, several solutions can be considered.

First we could choose for $\boldsymbol{P}^{j}$ some known constants, which is done in the classical approach (Section 2.1), or introduce constraints on the 3D point coordinates (for instance, we could expect points to lie on non-deformable solids). However, in this study, we seek to keep our system as flexible as possible. Consequently, we use a more generic approach, based on bundle adjustment [4]. To this aim, we work in the camera coordinate system, and simplify this system to ensure that matrices $\boldsymbol{P}^{j}$ only represent rotations and translation from camera to camera. Then, we find simultaneously $\boldsymbol{P}^{j}$ and $\boldsymbol{X}_{i}$ that minimize the reprojection error, i.e., the distance between 
the estimated point projection $\widehat{\boldsymbol{x}}_{i}^{j}$ and its observation $\boldsymbol{x}_{i}^{j}$ on the image. This leads to the following minimization problem

$$
\min _{\boldsymbol{X}_{i}, \boldsymbol{P}^{j}} \sum_{i, j}\left[\boldsymbol{x}_{i}^{j}-\widehat{\boldsymbol{x}}_{i}^{j}\left(\boldsymbol{X}_{i}, \boldsymbol{P}^{j}\right)\right]^{2} .
$$

Note that bundle adjustment is not convex and thus relies on a correct initialization. Moreover it requires a set of $2 \mathrm{D}$ observations and does not directly outputs the 3D coordinates of estimated points in the aircraft coordinate system. These issues have to be accounted in the method, and solutions are given through the processing chain.

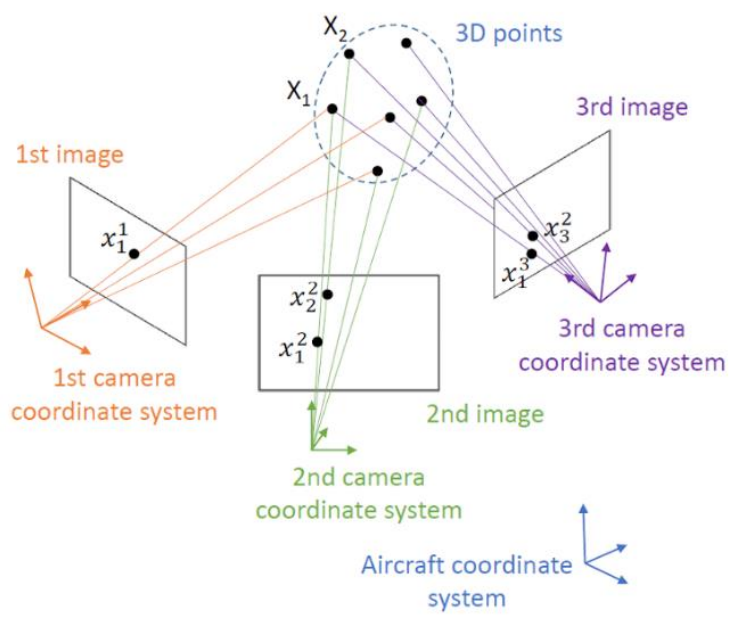

Fig. 5. Diagram of the multiple view photogrammetry for 3 cameras.

\subsection{Algorithmic Chain}

The algorithm chain is summarized as follows:

i. Camera and scene calibration

ii. Image acquisition and undistortion

iii. Observation detection

iv. Bundle Adjustment

v. Registration

vi. Parameters update

Step (i) is performed before flight and step (ii) to (v) are repeated for each new camera acquisition.

Step (i): The camera calibration is performed using pictures of a chessboard from different angles and distances, and using the OpenCV calibration functions [5], which outputs the intrinsic parameters of the cameras and distortion coefficients. The scene is then calibrated to initialize the positions of the 3D points and cameras. This step is conveniently performed using pictures from a drone and the photogrammetry software Metashape [6] (illustrated in Fig. 6). Note that a drone can take pictures of the full scene from a wide range of angles in less than 30 minutes.

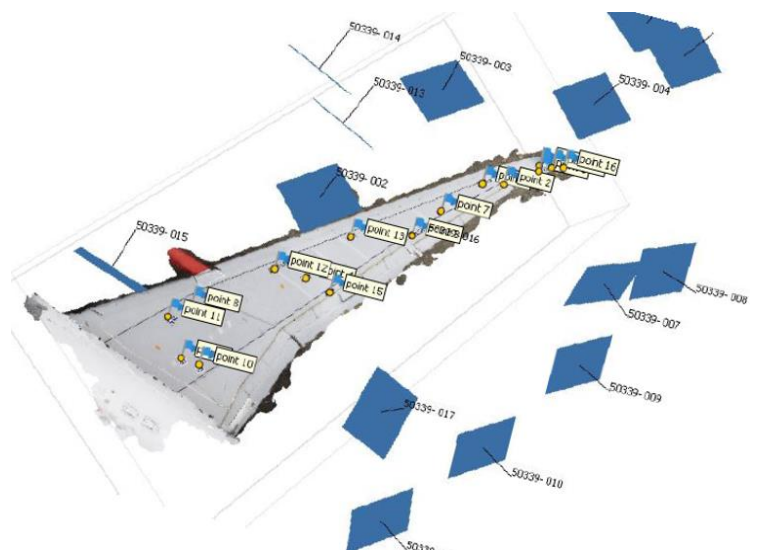

Fig. 6. Initial 3D reconstruction using the Metashape software and images from a drone. Images are displayed in blue at their point of view.

Step (ii): For each new image acquisition, we use the OpenCV undistortion function with the distortion parameters estimated in Step (i).

Step (iii): We propose three different ways to detect the 2D observation of the 3D points. First, we can use the natural features existing on the wing surface (corners, joints, writing, etc.). We find points with strong information using the ORB detection algorithm [7] and describe these points using a combination of ORB, SIFT [8][7] and DAISY [9] descriptors. Descriptors basically attribute a signature to a point based on the intensity values of it surrounding pixels. Descriptors are then compared between images to find matching points (see Fig. 7), which is performed in our case using the FLANN algorithm [10]. To increase the number of detected points, we use the guided matching algorithm described [11].

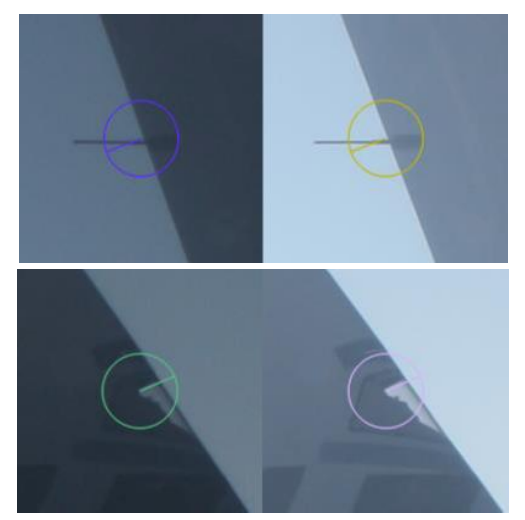

Fig. 7. Example of natural features of the wing detected and matched on a pair of images. Left and right images correspond to two distinct cameras.

The second approach to generate observations consists in exploiting the presence of black lanes 
along the wings of most aircraft. To do so, we rectify images using camera geometric properties estimated from a first set of corresponding points [14]. Image rectification allows the horizontally alignment of the corresponding points in a pair of images (see Fig. 8). Once images have been rectified, matching points can be found by searching lanes in image lines, which can be done using sub-pixel line detection methods [12][13].

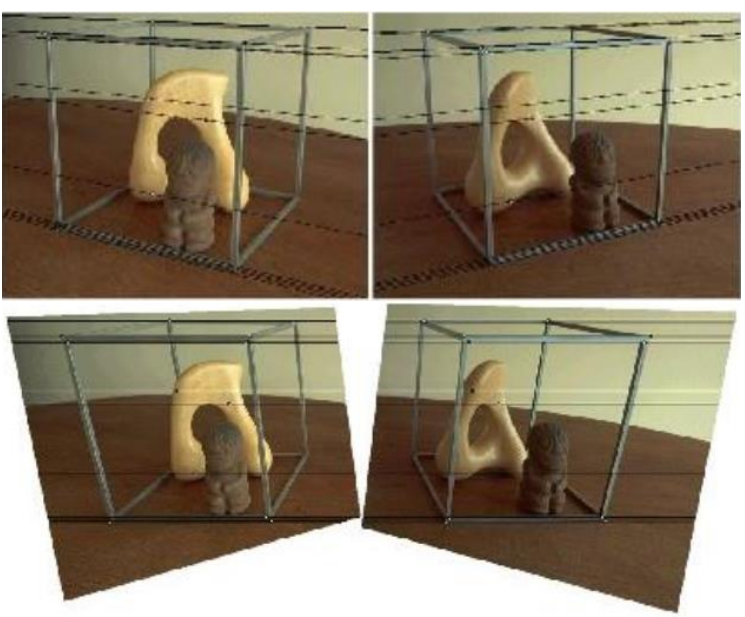

Fig. 8. Example of stereo image rectification (from [14] Zhang). Top - original pair of images, bottom - rectified images.

The third approach, when possible, is to use markers stuck on the wing surface. Markers allow an easy and accurate localization of the observations. A more detailed description of the markers is given in Section 4.1.

Step (iv): The initialization from step (i) and the set of 2D observation from step (iii) are used as input to the bundle adjustment, in order to minimize the reprojection error (1). The algorithm outputs the optimum 3D points $\boldsymbol{X}_{i}$ and the camera matrices $\boldsymbol{P}^{j}$ in the coordinate system of the cameras.

Step (v): The registration phase is performed using the rear camera. This camera can observe reference points close to the aircraft fuselage (see Fig. 9), which are considered as quasi-static during the flight. By detecting these points on the rear view image, we can recover the transfer matrix from the rear camera to the aircraft coordinate system. Since the rear camera is also part of bundle adjustment in Step (iv), we also know the transfer matrix from this camera to the global camera coordinate system. Finally these matrices are combined to transfer the 3D points and cameras to the aircraft coordinate system.

Step (vi): The initial parameters are updated, taking the output of Step (v) as knew initial positions of the algorithm.

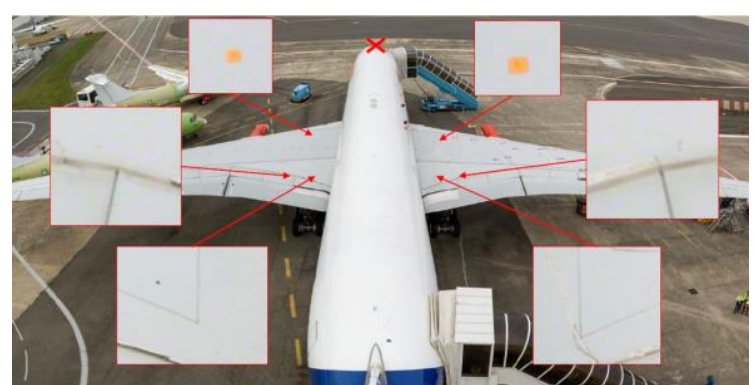

Fig. 9. Rear camera view with some reference points zoomed.

\subsection{Constrained Bundle Adjustment}

The method described in the previous section enables the 3D shape of the wing to be reconstructed, for any camera and marker motion, using no prior knowledge about wing deformations.

In this section, we go further and improve the performance of the method by introducing prior knowledge about the aircraft mechanical limits. We take benefit from the FEM of the wings to define limits corresponding to the ultimate loads. Assuming that the wing would break in such extreme cases, we consider that they are not possible during the tests. Thus, we construct a set of constraints that we inject in the classical bundle adjustment, such as local bending or elongation constraints (see Fig. 10). The resulting constrained bundle adjustment is constructed by adding a penalty term to the classical reprojection error.

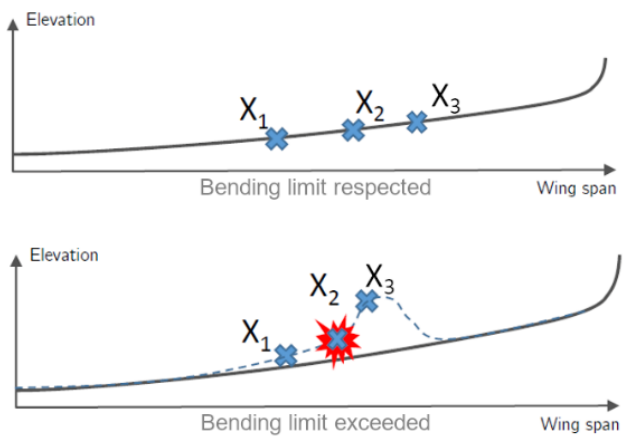

Fig. 10. Illustration of the local bending constraint: Top - constraints is respected, bottom - constraints is exceeded, and thus will be activated in the constrained bundle adjustment.

\section{Experimentations and Results}

This section first describes the system installation for flight and presents some results obtained from tests conducted on ground.

\subsection{Preparation for Flight Tests}

Our aim is to operate and validate our system on an A350-1000, whose wing is equipped with an IMU that will give a ground truth. 
The test was conducted using ruggedized cameras, offering a $31 \mathrm{MP}$ sensor with global shutter, while preserving small dimensions (about $60 \times 60 \times 60 \mathrm{~mm}$ ). Metrological windows were used instead of classical ones to prevent optical distortions. The shape of these camera, combined with our method, which is adapted to camera motions, allows an easy installation of the cameras on the aircraft window using simple 3D printed fixations. Cameras are placed as depicted in Fig. 11. Cameras 1 and 4 look at the wing end half, camera 2 and 3 look at the engine to tip section, and the rear camera 5 looks at the fuselage and the wing beginning.

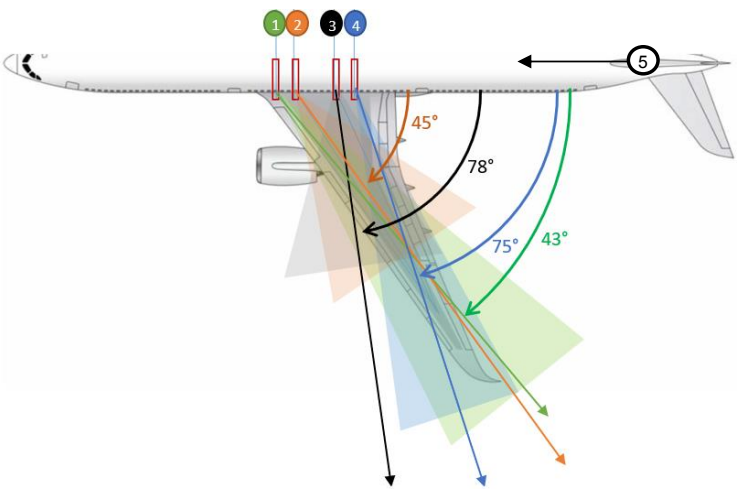

Fig. 11. Positions of the 5 cameras for the tests.

For the acquisition and data storage, dedicated computers were prepared with suited coaxlink cards and synchronization cards. During tests, data are recorded directly on the computer using the StreamPix software.

On the wing side, for robust and accurate detection, we use quadrant markers printed on stickers using matte ink. These markers can support flight conditions and the matte format allows correct detection for any illumination. Elongated stickers of various sizes were used depending on their positions onto the wing to ensure a similar aspect ratio when seen by the cameras (see Fig. 12 and Fig. 15). The detection can reach sub-pixel accuracy [16].

This setup was used in different experiments conducted on ground to evaluate the relevance of the proposed system.

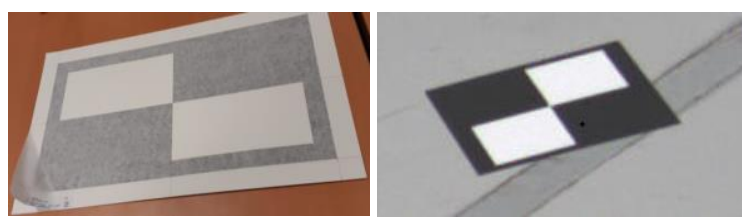

Fig. 12. Stickers of quadrant markers. Left - close view before installation, right - aspect of the target from camera view.

\subsection{Test on Ground}

The new photogrammetry system was evaluated on ground on an A350-900. The devices were installed in order to reproduce in-flight installation. We installed our markers on the wing surface, plus tape graduations on the two black lanes of the wing, at about every $30 \mathrm{~cm}$ (shown in Fig. 13), and four $4 \mathrm{~K}$ cameras on an aircraft window. The cameras were placed about one meter above the windows to produce similar views that would be obtained at a window position with the wing bent (see Fig. 14). Examples of the acquired views are displayed in Fig. 15. Note that the wing and the cameras were not moving during this test. However, several pictures of the wing were acquired during three days to see the impact of the luminosity on the reconstruction. As expected, we monitored illumination variations and moving reflections on the wing, as visible in Fig. 15 for camera 3. This induced the failure of correct detection of the graduation located at the wing end. However, quadrant markers remained always detectable.
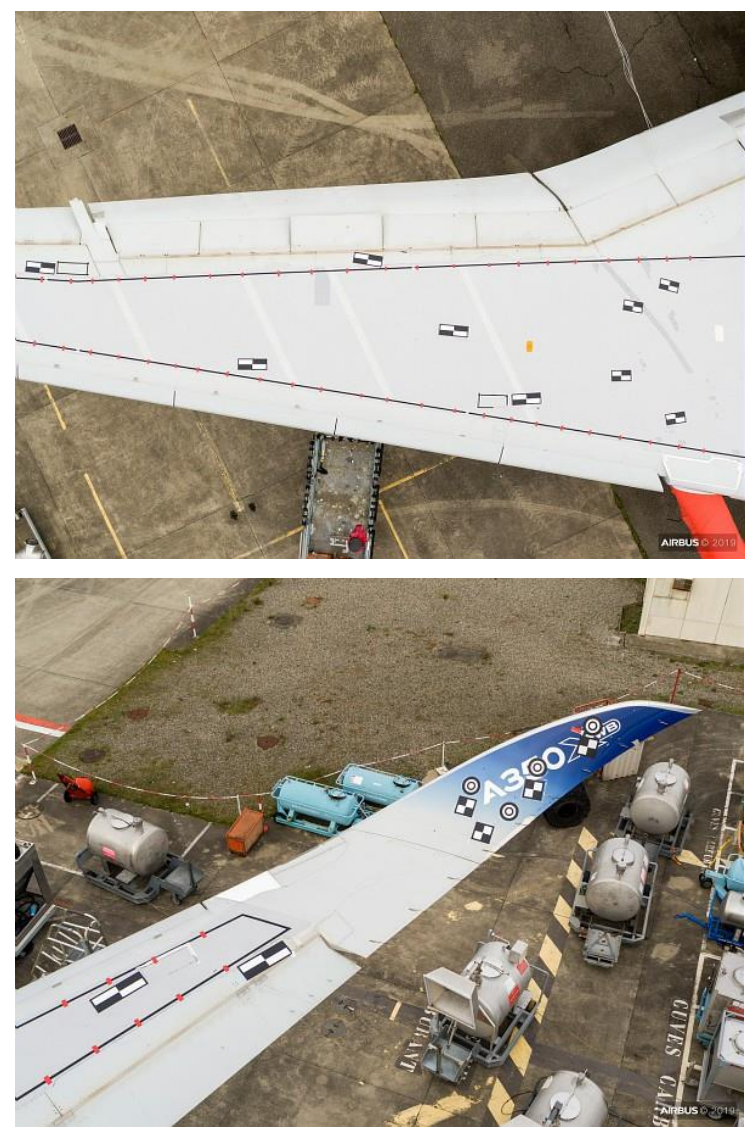

Fig. 13. Markers and graduations installed on the A350-900 wing.

To initialize the algorithm presented in Section 3.2 , we triangulated the $3 \mathrm{D}$ positions of reference points on the wing, using images taken from a drone (see Fig. 6). We shook manually 
the wing at its tip to produce vibrations confirming the behavior of the system for small deformations. The amplitude of the vibrations was estimated to about $5 \mathrm{~cm}$ using a scale board.

The proposed algorithm was implemented in Python and tested on different images. Quadrant markers where detected and their 3D coordinates were estimated and registered in the Aircraft coordinate system. Fig. 16 shows the results of the algorithm superimposed on the reference $3 \mathrm{D}$ shape of the wing (on ground). $A$ ground truth was not available for this test and therefore we cannot determine the accuracy of the reconstruction. However, the results seem to correctly coincide with the $3 \mathrm{D}$ reference shape.

The study of mechanical limits applied as constraints on the bundle adjustment has also shown promising results. Fig. 17 displays some reconstruction results for markers with and without a volume constraint. This constraint states that points in the aircraft coordinate system can move only in a cylinder with a $5 \mathrm{~cm}$ radius, oriented towards the $z$ axis. Using the volume con- straint allows us to obtain estimated displacements of the wing tip close to $5 \mathrm{~cm}$, which is coherent with our observation using the scale board.

\section{Conclusion}

This paper presented a new photogrammetry method for estimating a wing deformation. This method bypasses some limitations of previous approaches, offering easy installation, more flexibility, dynamic mapping, with no dedicated test, and delivering more accurate estimations of the wing deformations. The proposed method is a flexible adaptation of the classical multiple view photogrammetry for the specific case of 3D deformation estimation. Our algorithm goes even further by introducing mechanical limits in the process to improve the reconstruction accuracy. The system has been prepared for flight tests and tested on ground, where experimentations delivered promising results. The next step will be to evaluate the system in flight with countermeasure. Future work will focus on the detailed assessment of the metrological accuracy of the system as a real sensor.
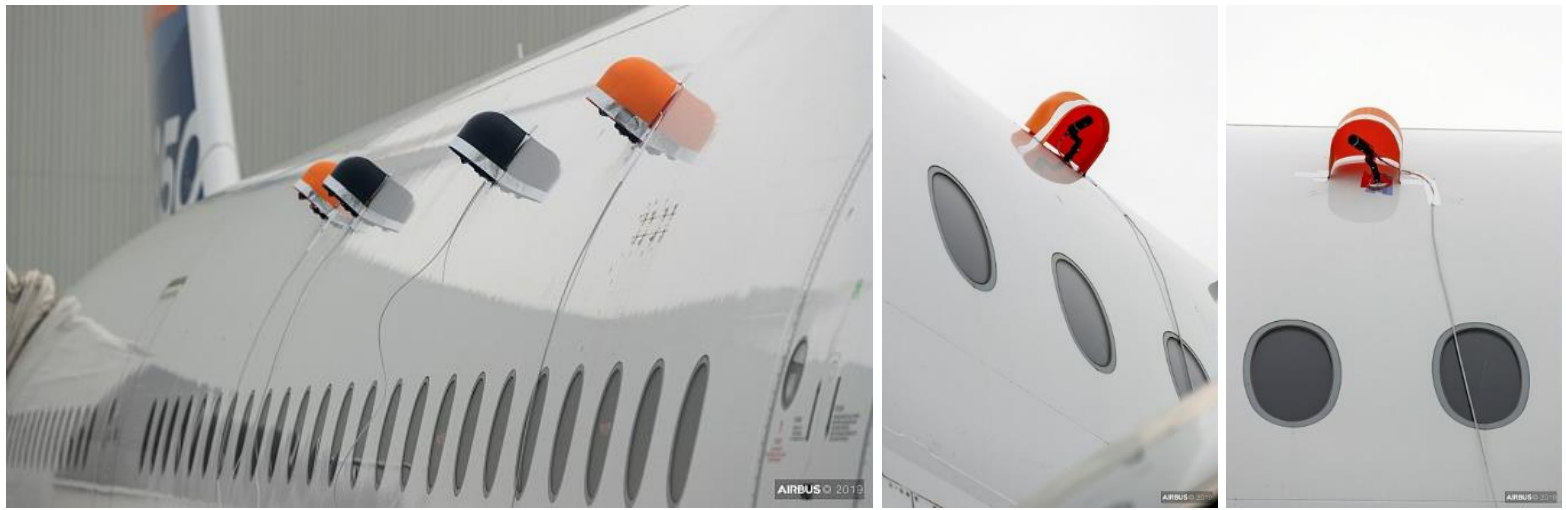

Fig. 14. Camera installation above windows of an A350-900.
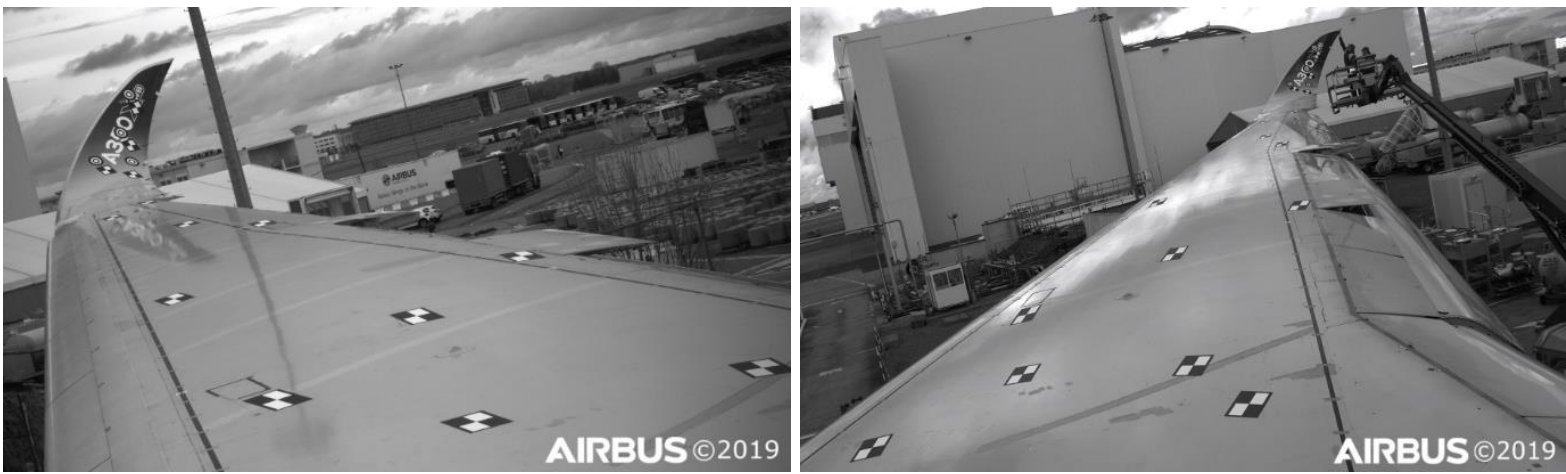

Fig. 15. Example of views from the camera during the test on ground. Left - camera 1, right- camera 3. 


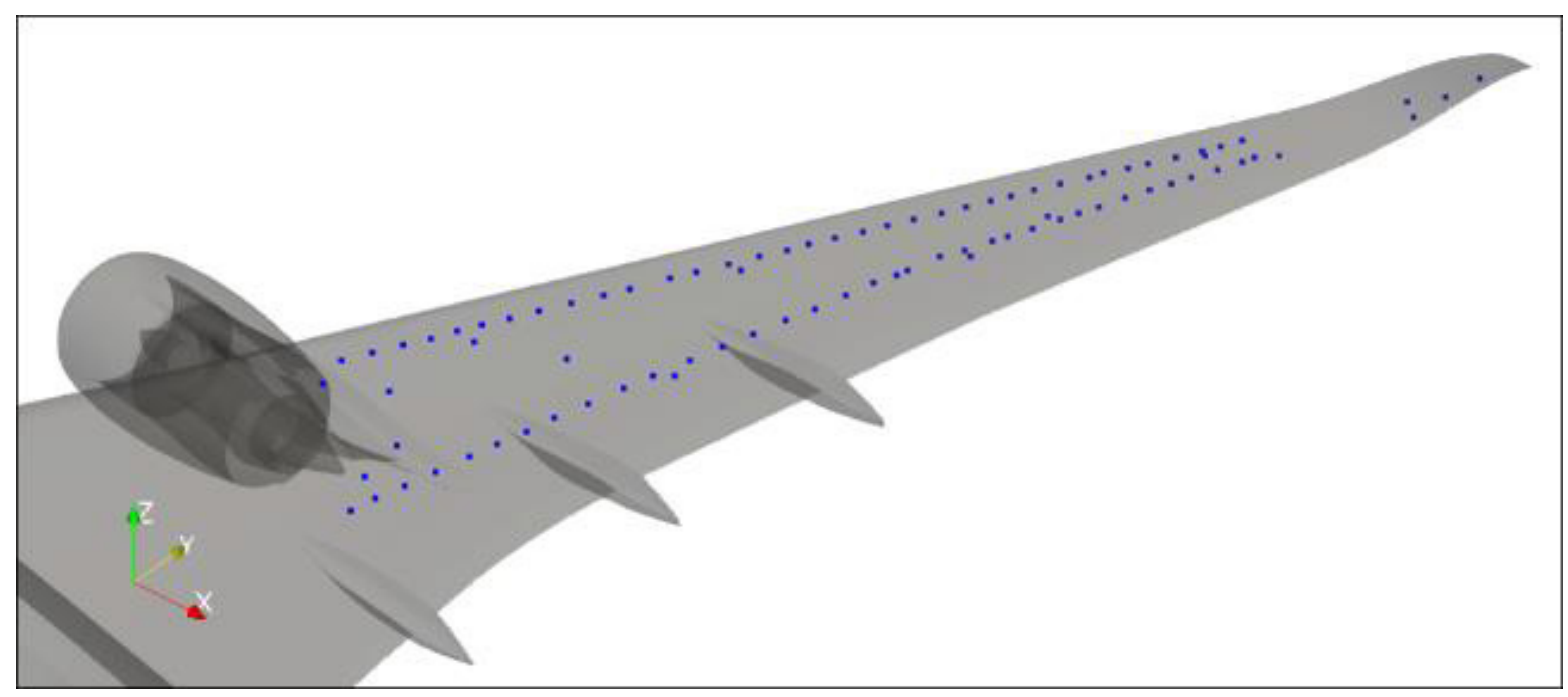

Fig. 16. Results obtained with the proposed algorithm. Blue dots indicate the $3 D$ reconstructions of graduations and markers located on the A350-900 wing (that have been superimposed on the 3D shape reference of the wing).
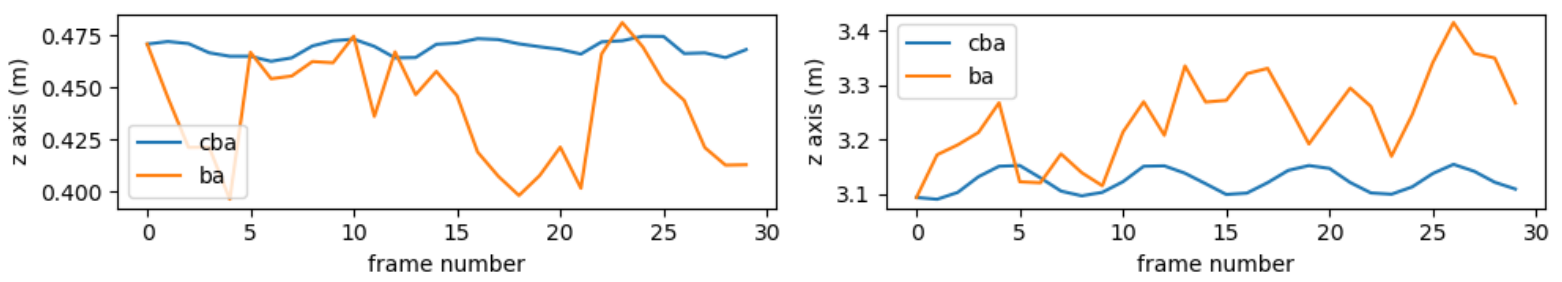

Fig. 17. Estimated bending of two markers versus time while the wing was shaken. The result of the algorithm without constraint is displayed in blue (ba) and the result with a displacement constraint is displayed in orange (cba). Left - marker at the middle of the wing, right - marker on the wing tip.

\section{References}

[1] D. Barrows, "Videogrammetric model deformation measurement technique for wind tunnel applications," in Proc. 45th AIAA Aerospace Sciences Meeting and Exhibit, Reno, Nev., USA, Jan. 2007; doi: 10.2514/6.2007-1163

[2] T. Liu, A. W. Burner, T. W. Jones, and D. A. Barrows, "Photogrammetric techniques for aerospace applications," Progress in Aerospace Sciences, vol. 54, pp. 1-58, Oct. 2012; doi: 10.1016/j.paerosci.2012.03.002

[3] "Airbus BLADE" laminar flow wing demonstrator makes first flight" (Press release). Airbus, 26 Sep 2017. Available at: https://www.airbus.com/newsroom/press-releases/en/2017/09/airbus_blade_laminar-flow-wing-demonstrator-makesfirst-fligh.html

[4] R. Hartley and A. Zisserman, Multiple View Geometry in Computer Vision, Cambridge University Press, 2004; isbn: 978-0-511-18618-9

[5] Intel, The OpenCV Reference Manual, 2.4.13.7 edition, Dec. 2019; See http://opencv.org/

[6] Agisoft LLC, St. Petersburg, Russia, Agisoft Metashape User Manual: Professional Edition, Version 1.6, 2020, available at:

https://www.agisoft.com/downloads/user-manuals/.
[7] E. Rublee, V. Rabaud, K. Konolige, and G. Bradski, "ORB: An efficient alternative to SIFT or SURF," in Proc. Int. Conf. Computer Vision, Barcelona, Spain, Nov. 2011; doi: 10.1109/iccv.2011.6126544

[8] D. G. Lowe, "Distinctive image features from scale-invariant keypoints," Int. J. Comput. Vis., vol. 60 , pp. 91-110, Nov. 2004; doi: 10.1023/b:visi.0000029664.99615.94

[9] E. Tola, V. Lepetit, and P. Fua, "DAISY: An efficient dense descriptor applied to wide-baseline stereo," Trans. Pattern Anal. Machine Intell., vol. 32, pp. 815-830, May. 2010; doi: 10.1109/tpami.2009.77

[10] M. Muja and D. Lowe, "Fast approximate nearest neighbors with automatic algorithm configuration," in Proc. Int. Conf. Comput. Vis. Theory and Applications (VISAPP), Lisboa, Portugal, Jan. 2009; doi: 10.5220/0001787803310340

[11] R. Shah, V. Srivastava, and P.J. Narayanan, "Geometry-aware feature matching for structure from motion applications," in Proc. Conf. on Applications of Computer Vision, Waikoloa, HI, USA, Jan. 2015; doi: 10.1109/wacv.2015.44

[12] R. G. Gioi, J. Jakubowicz, J. M. Morel, and G. Randall, "LSD: a line segment detector," Image 
Processing on Line, vol. 2, pp. 35-55, Mar. 2012; doi: 10.5201/ipol.2012.gjmr-lsd

[13] R. G. Gioi and G. Randall, "A sub-pixel edge detector: an implementation of the canny/devernay algorithm," Image Processing on Line, vol. 7, pp. 347-372, Nov. 2017; doi: 10.5201/ipol.2017.216

[14] C. Loop and Z. Zhang, "Computing rectifying homographies for stereo vision," in Proc. Computer Society Conf. on Computer Vision and Pattern Recognition, Fort Collins, Co., USA, Jun. 1999; doi: 10.1109/cvpr.1999.786928
[15] F. Boden, N. Lawson, H. W. Jentink, and J. Kompenhans, Advanced In-Flight Measurement Techniques, Springer Berlin Heidelberg, 2013; isbn: 978-3-642-34738-2

[16] A. M. G. Tommaselli and A. Berveglieri, "Measuring photogrammetric control targets in low contrast images," Bulletin of Geodetic Sciences, vol. 24, pp. 171-185, Jun. 2018; Doi: 10.1590/s198221702018000200012 